\title{
Clinico Histopathological Correlation in Leprosy
}

\author{
Mridula Pasupathy ${ }^{1}$, Ramesh Bhat $\mathrm{M}^{2}$ \\ ${ }^{I}$ Department of Dermatology, Fr.Muller Medical College Hospital, India \\ ${ }^{2}$ Department of Dermatology, Fr.Muller Medical College Hospital, India
}

\begin{abstract}
:
Introduction: Leprosy is a chronic, infectious disease caused by Mycobacterium leprae. It is classified into five groups based on clinical, histological, microbiological and immunological criteria (Ridley \& Jopling Classification). However a great variation is seen in interpretation of clinical and histopathological examination of these lesions. The present research was taken to study the correlations between the clinical and histological diagnosis and to evaluate the importance of skin biopsy as an important diagnostic and spectrum defining tool.

Methodology: A retrospective Hospital-based study was conducted in patients of Leprosy, who attended Dermatology Out Patient Department for a period of 18 months. Clinical diagnosis was noted and the biopsies were processed as per standard protocol in the Department of Pathology. The clinical and histopathological concordance was calculated using percentage parity.

Results \& Conclusion: In a total of 50 cases, 28 (56\%) males and 22 (44\%) females .The histopathological diagnoses from our study showed agreement with clinical diagnoses in 25 (50\%) cases. The maximum concordance of $80 \%$ was seen in LL cases followed by TT (57\%), BT(53.33\%), IDL (50\%), BL (33.33\%) and major discordance was observed in BB(14.29\%) cases.
\end{abstract}

Keywords: clinico-histopathological, mycobacterium leprae, fite faraco, acid fast bacilli, granulomas

\section{Introduction}

Leprosy is a chronic, infectious disease caused by Mycobacterium Leprae presses itself in different clinico-pathological forms, depending on the immune status of the host. ${ }^{1}$ Before confirming a case of Leprosy of particular type, the clinical features should be correlated and confirmed with histological examination along with bacteriological index and start the multidrug treatment. ${ }^{2}$ The disease spectrum has been characterised in a number of classification systems, most widely being the Ridley-Jopling classification. In this classification, leprosy has been divided into five groups as Tuberculoid (TT), Borderline tuberculoid(BT),Mid-borderline (BB), Borderline Lepromatous(BL), and Lepromatous (LL) ${ }^{3}$ Though the clinical diagnosis is based on characteristic skin lesions with sensory loss, a great variation is seen in interpretation of these lesions, both clinically and histopathologically. ${ }^{4}$ The current study was undertaken to correlate different clinical types of leprosy and histopathology of skin biopsies.

\section{Materials And Methods}

A retrospective Hospital-based study was conducted in 50 patients of Leprosy, who attended Dermatology Out Patient Department, Father Muller Medical College Hospital, Mangalore between December 2014 and May 2016 (i.e., 18 months). The spectrum of the disease is diagnosed clinically and graded into Tuberculoid Type (TT), Borderline Tuberculoid (BT), Mid-borderline (BB), Borderline Lepromatous (BL), Lepromatous Leprosy (LL) and Indeterminate type (IDL) as per Ridley-Jopling classification which is accepted worldwide. 8 Cases were selected regardless of their age, sex, socioeconomic status and occupation. Patients already treated with anti-leprosy medications in any time earlier were excluded. Skin biopsies were taken from active lesions in all patients after taking informed consent and approval from institutional ethical committee and biopsies were processed as per standard protocol in Department of Pathology. They were stained by Hematoxylin \& Eosin stain. AFB were demonstrated using Fite Faraco stain. The clinical and histopathological correlation was calculated using percentage parity.

Statistical Analysis

Statistical analysis is done using range, frequency, percentage, chi square test.

\section{Results}

The study group consisted of total 50 patients, out of which 28 (56\%) males and 22 (44\%) females between 8 and 86 years of age.(Table 2). The age group of the patients ranged from 10 years to 74 years. The majority of the cases belonged to the age group of 31-50 years i.e., 27 (54\%) cases .(Table 2) Clinically, BT was 
the most common type of leprosy with $30 \%$ (15) followed by TT in $28 \%(14)$, BB in 14\%(7), IDL in 12\%(6) LL in $10 \%(5)$ and BL in 6\%(3). Histopathologically, majority of the cases i.e., 36\%(18) belonged to TT followed by BT in $28 \%(14)$, LL in 12\%(6),IDL in $10 \%(5), \mathrm{BL}$ in $8 \%$ (4) and BB in 6\%(3) (Table 3)

\section{Discussion}

Leprosy is a chronic, infectious disease caused by Mycobacterium leprae and is present in different clinico-pathological forms, depending on the immune status of the host. The study of pathological changes in leprosy lesions has contributed a great deal to understanding the disease and clinico-pathological correlative

studies have provided further insights into the disease, its varied manifestations and complications. ${ }^{5}$ Accurate classification of leprosy is needed as it is present in different clinicopathological forms. The most widely accepted classification system is that of Ridley- Jopling. ${ }^{3}$ However, many diversities are seen between the histopathological and clinical features. The patients in our study were $28(56 \%)$ males and $22(44 \%)$ females between 8 and 86 years of age. The majority of patients $27(54 \%)$ were in the age group of 31-50 years . A similar study conducted by Moorthy et al. from India, among 372 leprosy patients showed $242(65.05 \%)$ males and $130(34.95 \%)$ females between three and 86 years of age. The majority of patients were in the age group of $20-29$ years $(20.70 \%)$ and least affected were children below 9 years $(6.45 \%){ }^{6}$

In our study ,clinically, BT was the most common type of leprosy with $30 \%$ (15) followed by TT in $28 \%(14)$, BB in 14\%(7), IDL in 12\%(6) LL in 10\%(5) and BL in 6\%(3). Histopathologically, majority of the cases i.e., $36 \%(18$ ) belonged to TT followed by BT in $28 \%(14)$, LL in 12\%(6),IDL in $10 \%(5)$,BL in $8 \%$ (4) and $\mathrm{BB}$ in 6\%(3). Overall agreement in the diagnosis was seen in $25(50 \%)$ cases.

The maximum concordance of $80 \%$ was seen in LL cases followed by TT (57\%), BT(53.33\%), IDL $(50 \%), \mathrm{BL}(33.33 \%)$ and major discordance was observed in $\mathrm{BB}(14.29 \%)$ cases. Maximum concordance was observed in LL type of leprosy, which was similar in studies by Mathur MC et al., Giridhar M et al and Moorthy et al.However, concordance differed variably when compared with other types of Leprosy, which may be due to more precise diagnostic criteria laid down in histopathology with emerging microbiological and immunological techniques. The observations strongly suggest the importance of histopathological diagnosis in these cases, as lesions are easy to diagnose clinically towards Lepromatous pole of the disease. ${ }^{7}$ Thus, skin biopsies should be taken from the representative lesions so that the diagnosis can be established as an adjunct to clinical diagnosis and fulfilling the criteria for classifying the disease spectrum, which helps in proper treatment and eradication of the disease. Considering the data of present study and other comparative studies, we may state that maximum correlation is seen with LL as it shows a fixed histopathology. However, in early cases of midborderline (BB) and borderline lepromatous (BL) forms of disease, histopathology shows some ambiguity.

Table 1. Showing age distribution in the subjects

\begin{tabular}{|c|c|c|}
\hline $\begin{array}{l}\text { Age } \\
\text { group(years) }\end{array}$ & $\begin{array}{l}\text { No of } \\
\text { cases }\end{array}$ & Percentage \\
\hline$<30$ years & 18 & $36 \%$ \\
\hline 31-50 years & 27 & $54 \%$ \\
\hline$>50$ years & 5 & $10 \%$ \\
\hline Total & 50 & $100 \%$ \\
\hline
\end{tabular}

Table 2. Showing frequency and percentage of gender distribution

\begin{tabular}{|l|l|l|}
\hline & Frequency & \% age \\
\hline Females & 22 & $44 \%$ \\
\hline Males & 28 & $56 \%$ \\
\hline Total & 50 & 100 \\
\hline
\end{tabular}

Table 3. Showing clinical and histopathological distribution of leprosy

\begin{tabular}{|l|l|l|l|l|}
\hline $\begin{array}{l}\text { Type of } \\
\text { leprosy }\end{array}$ & $\begin{array}{l}\text { No of } \\
\text { clinical } \\
\text { cases }\end{array}$ & $\begin{array}{l}\text { \% of } \\
\text { clinical } \\
\text { cases }\end{array}$ & $\begin{array}{l}\text { No of histopathological } \\
\text { cases }\end{array}$ & $\begin{array}{l}\% \text { of histopathological } \\
\text { cases }\end{array}$ \\
\hline BB & 7 & 14 & 3 & 6 \\
\hline BL & 3 & 6 & 4 & 8 \\
\hline BT & 15 & 30 & 14 & 28 \\
\hline IDL & 6 & 12 & 5 & 10 \\
\hline LL & 5 & 10 & 6 & 12 \\
\hline TT & 14 & 28 & 18 & 36 \\
\hline Total & 50 & 100 & 50 & 100 \\
\hline
\end{tabular}


Table 4. AFB positivity in various types of leprosy

\begin{tabular}{|l|l|l|l|}
\hline \multirow{2}{*}{ Type of leprosy } & \multicolumn{2}{|l|}{ AFB POSITIVITY } & Percentage positive \\
\cline { 2 - 4 } & NUMBER POSITIVE & 33.30 \\
\hline & BB & 1 & 25.00 \\
\hline & BL & 1 & 28.60 \\
\cline { 2 - 5 } & BT & 4 & - \\
\hline & IDL & 0 & 66.70 \\
\cline { 2 - 4 } & LL & 4 & - \\
\hline & TT & 0 & 20.00 \\
\hline Total & & 10 & \\
\hline
\end{tabular}

Table 5. Clinico histopathological concordance in leprosy

\begin{tabular}{|c|c|c|c|c|c|c|c|c|c|}
\hline & & \multicolumn{6}{|c|}{$\begin{array}{l}\text { Histopathological break up among clinically diagnosed } \\
\text { cases }\end{array}$} & Total & \multirow[b]{2}{*}{$\%$ parity } \\
\hline & & BB & $\mathrm{BL}$ & $\mathrm{BT}$ & IDL & LL & $\mathrm{TT}$ & $\begin{array}{l}\text { No of clinical } \\
\text { cases }\end{array}$ & \\
\hline \multirow{7}{*}{$\begin{array}{l}\text { clinical } \\
\text { type }\end{array}$} & BB & 1 & 1 & 0 & 0 & 1 & 4 & 7 & $14.29 \%$ \\
\hline & $\mathrm{BL}$ & 1 & 1 & 1 & 0 & 0 & 0 & 3 & $33.33 \%$ \\
\hline & $\mathrm{BT}$ & 1 & 1 & 8 & 0 & 1 & 4 & 15 & $53.33 \%$ \\
\hline & IDL & 0 & 0 & 1 & 3 & 0 & 2 & 6 & $50.00 \%$ \\
\hline & LL & 0 & 1 & 0 & 0 & 4 & 0 & 5 & $80.00 \%$ \\
\hline & TT & 0 & 0 & 4 & 2 & 0 & 8 & 14 & $57.14 \%$ \\
\hline & Total & 3 & 4 & 14 & 5 & 6 & 18 & 50 & $50.00 \%$ \\
\hline
\end{tabular}

p $<0.05$, significant

\section{Conclusion}

Leprosy is a chronic granulomatous disease widely prevalent in India and is present in different clinicopathological forms. Study of these lesions has contributed a great deal in understanding the disease. Most cases can be diagnosed clinically; especially those towards the Lepromatous pole of the disease, however, other types of Leprosy pose a significant problem in clinical diagnosis. Skin biopsy is a useful tool in confirming the clinical diagnosis of leprosy as well as for the therapeutic guide.

\section{Acknowledgement}

I acknowledge the support and help of Dr. Ramesh Bhat, Dr. Jacintha Martis, Dr. Sukumar D, Department of Dermatology, Dept of Pathology, MRD staff, Mrs. Sucharitha (for statistical support) and my colleagues, Father Muller Medical College, Mangalore, India.

\section{Conflict Of Interest}

None.

\section{References}

[1]. Abulafia J, Vignale RA. Leprosy: Pathogenesis updated. Int J Dermatol 1999;38:321-334.

[2]. Nitesh Mohan, Nitin Mishra. Clinico Histopathological Correlation Within The Spectrum Of Hansen's Disease: A Multicentric Study In North India. Int J Med Res Health Sci 2013; 2(4):887-92.

[3]. Mathur MC, Ghimire RBK, Shrestha P,Kedia SK. Clinico histopathological Correlation in Leprosy. Kathmandu Univ Med J. 2011;36(4):248-51.

[4]. Giridhar M, Arora G, Lajpal K, Chahal KS. Clinicohistopathological concordance in Leprosy- A Clinical, Histopathological and Bacteriological study of 100 cases. Indian J Lepr 2012;84:217-25.

[5]. Kalla G, Salodkar A and Kachhawa D (2000).Clinical and histopathological correlation in leprosy. Int J Lepr. 68: 184-185.

[6]. Moorthy BN, Kumar P, Chatura KR et al. Histopathological correlation of skin biopsies in leprosy. IJDVL 2001;67:299-301.

[7]. Vargas-Ocampo, Fransisco. Analysis of 6000 Skin Biopsies of the National Leprosy Control Program in Mexico. Int J Lepr Other Mycobact Dis. 2004;59:28-35.

[8]. Jopling WH, Mc Dougall. The disease. In: Jopling WH, Mc Dougall. (Authors) Handbook of leprosy. Fifth edition. CBS Publishers and distributors (India)2008;10-53. 